\title{
DYNAMICS OF FACIAL EXPRESSION: NORMATIVE CHARACTERISTICS AND INDIVIDUAL DIFFERENCES
}

\author{
K. L. Schmidt, J. F. Cohn \\ University of Pittsburgh \\ 4015 O'Hara St. \\ Pittsburgh PA 15260 USA
}

\begin{abstract}
Although the importance of facial expression in human computer interaction and in normal human interaction is widely acknowledged, there is very little data on the normative characteristics and stable individual differences for even the most common facial expressions. Dynamic characteristics of 195 spontaneous smiles from 95 individuals were measured using the facial action coding system, automated facial analysis and facial electromyography. Normative patterns observed included the characteristic timing of other facial actions with respect to action unit 12 ("smile") and a mean duration of 15.7 frames for smile onset. Stable inter-individual differences included patterns of nonverbal actions associated with individuals' smiles, and the amount of activity in the zygomaticus major muscle in two sessions recorded a year apart. These data are important in quantifying and fully describing individual differences in naturalistic human facial expression, as well as adding to our knowledge of spontaneous human smiles.
\end{abstract}

\section{INTRODUCTION}

The dynamic properties of spontaneous human facial expressions are of interest to both psychologists and computer scientists seeking to describe and replicate naturalistic interactive behavior. There is relatively little information available, however, on the normative qualities of facial expressions (but see [1][2]). For example, the smile (action unit 12, FACS Manual [5]) has been described as ballistic, with characteristic onset, peak and offset duration, but this finding has not been replicated in the literature [1]. Qualitative studies of the presence or absence of accompanying action units that change the appearance of the smile led Ekman [3] to identify 18 different types of smiles, but the dynamic relationships among these different facial movements have not been described for each smile type. Smile types are believed to correspond to situations eliciting different kinds of emotion. For example, the smile of enjoyment is distinguished from a social, polite smile by the addition of muscle activity visible in movement of the skin in the upper cheek and around the side of the eye (AU 6). The enjoyment or Duchenne smile (AU 12 with AU 6) and the social or non-Duchenne smile (AU 12 without AU 6) are observable in different contexts and under different emotional conditions. In unpleasant contexts, experienced emotion and cultural display rules combine to produce smiles such as the miserable or dampened smile (AU 12 with AU 15). It is unknown whether dampened smiles, however, are exclusive to the contexts in which they have been described. The frequency of smile types in everyday situations, and possible individual differences in the display of different action units is unknown.

There is even less information about potential individual differences in facial expression. Within populations, we know that there are differences in smiling among men and women, suggesting that women are more expressive than men on average [4]. At the individual level, there may also be stable patterns of facial expression that provide additional identifying information when combined with other biometrics. In this paper, we describe normative properties of spontaneous smiles, as well as explore the possibility of stable inter-individual differences in smiles.

\section{METHODS}

\subsection{Data Collection}

Original data were collected from a sample of subjects in a psychophysiological study of emotion. Manual facial action coding (FACS) [5], automated facial analysis using feature tracking (AFA) [6], and facial electromyography (EMG), were used to investigate the dynamic properties of smiling. Video and EMG data were collected throughout a session that included baseline (seated with eyes open) and viewing of film clips. Smiles studied were observed during the viewing of a comedy routine, and visual data (FACS and AFA) were collected in the period following each of the first three jokes. EMG data were collected for the duration of the session, including a baseline portion and the entire comedy routine ( $\sim 5$ minutes).

\subsection{Facial Expression Coding}

\subsubsection{Facial Action Coding System}

Smiles ( $n=195$ from 94 subjects) were manually coded using the Facial Action Coding System (FACS [5], and the presence of 
other facial actions overlapping with AU 12 (zygomaticus major "smile") were recorded. The order of action units occurring was recorded, along with the number of frames separating AU 12 and the nearest AU that was continuous with it for part or all of the smile duration. Action units occurring within 2 frames of AU 12 were coded as simultaneous, while those occurring $>3$ frames after AU 12 were coded as following, and those $>3$ frames before coded as preceding. Agreement between two certified FACS coders for the presence or absence of action units $6,12,14,15$, 17 , and 23 during smiles was 0.92 ( $\mathrm{n}=27$ smiles). Agreement for the order of action unit appearance was 0.86. Appearance of rigid head movements (AUs 51-58) and their relationship to AU 12 were also coded.

\subsubsection{Automated Facial Analysis}

Videotaped smiles were digitized at 30 frames per second and Automated Facial Analysis [8] using feature point tracking was performed on 50 of 195 smiles in this sample. The position of lip corners in a video series was recorded for the duration of $\mathrm{AU}$ 12 in the smile, and the longest continuous sequence of rising values in $r$ (polar coordinate of lip corner position) was recorded. This sequence of rising values was designated as the smile onset (see Figure 1. for an example).

\subsection{Facial Electromyography}

Facial electromyographic (EMG) data were collected using standard placement of electrodes over the zygomaticus major muscle [9], digitized at $256 \mathrm{~Hz}$, band pass filtered between 5 and $90 \mathrm{~Hz}$, and down-sampled at 1/30 second intervals, to correspond with the length of a video frame. EMG values reported here are $\mathrm{z}$ scores for zygomaticus major activity obtained during the comedy film clip, as compared to the mean values for that muscle during an eyes open baseline period. Values for a second identical session one year later are also reported. This group included subjects with video data $(\mathrm{n}=31)$ and additional subjects from the same study $(\mathrm{n}=35)$.

\section{NORMATIVE CHARACTERISTICS}

\subsection{Order of Appearance of Facial Actions}

Action units continuous with AU 12 were typical of those in the smiles described by Ekman (1985) for the 18 types of smiles, and include AU 6 (cheek raiser), AU 14 (dimpler), AU 15 (lip corner depressor), AU 17 (chin raiser), and AU 24 (lip pressor), among others [3]. Approximately one third of smiles consisted of AU 12 without other facial actions; remaining smiles had one or more action units co-occurring with AU 12.

The dynamic relationship among action units in a facial display has previously been reported only for the facial expression known as "brow raise" (AU 1+AU 2). In this spontaneous greeting expression, the appearance of other action units is typically suppressed during the duration of the expression [2]. In contrast, during spontaneous smiles, we found that other action units closely followed the appearance of AU 12. The appearance of AU 12 was either simultaneous with or closely followed by one or more associated action units, such as AU 6, AU 15 or AU 17 ( $n=117 ; 88 \%)$. AU 6 was the first action unit to follow AU 12 in $62 / 133$ or $47 \%$ of all smiles with multiple action units. For these smiles, AU 6 appeared an average of 11 frames after the beginning of the smile (see example in Figure 1.)

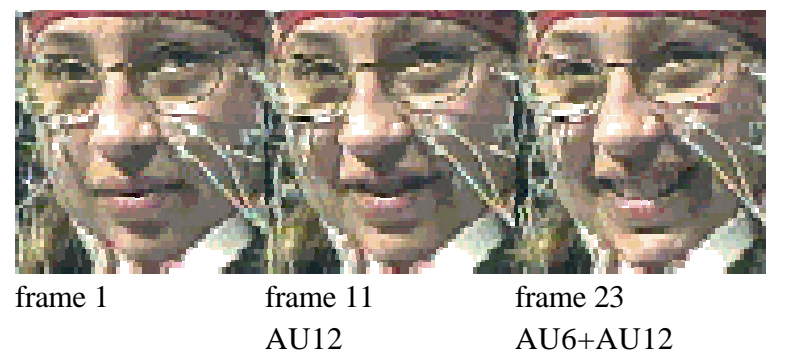

change in position of

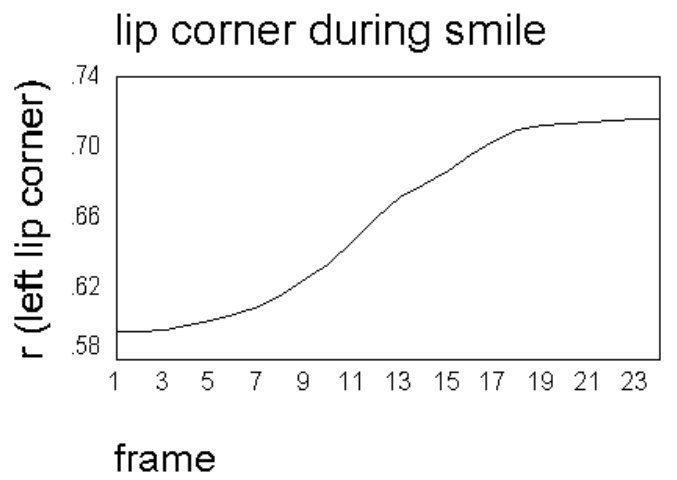

Figure 1. Appearance of action units during smile onset in a Duchenne smile

Although action units 14, 15 and 17, components of the dampened smile, were not predicted to occur during the viewing of a comedy routine, 31 smiles of this type were also observed (see Figure 2. for an example). In 25 of these smiles, the dampening action occurred either simultaneously with, or following AU 12.

\subsection{Smile onset duration}

Average duration of increasing lip corner height and width was 15.7 frames (SD 8.4) measured using automated facial analysis for 50 of the 195 manually coded smiles. Onset duration was rapid, and relatively consistent across individuals $(n=50)$ in this sample, as predicted for spontaneous smiles (see Figures 1. and 2.). The approximately half second duration of smile onset is consistent with overall duration of $0.67-4.00$ seconds previously reported 


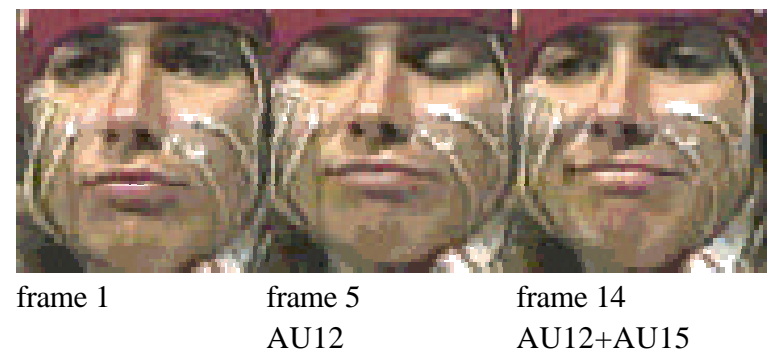

change in position of

lip corner during smile

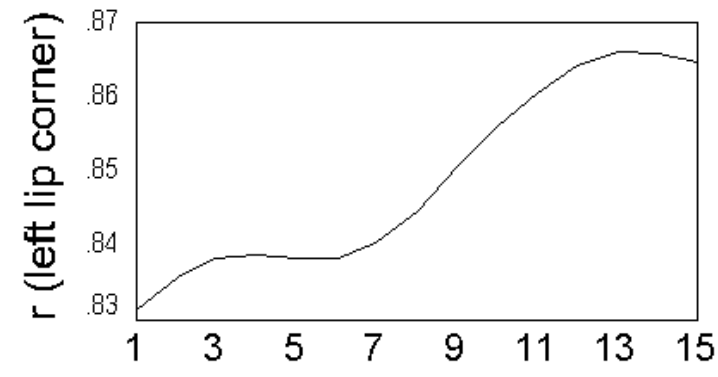

frame

Figure 2. Appearance of action units during smile onset in a dampened smile

for spontaneous smiles, using manually coded data [1]. It is also fairly consistent across different smile types (Duchenne, nonDuchenne, dampened) in this sample.

\section{INDIVIDUAL DIFFERENCES}

\subsection{Consistency in Facial Actions over time}

Of the individuals with 3 smiles $(n=45), 64 \%$ consistently displayed a specific combination of action units across all three smiles (see Table 1.)

Table 1. Individually consistent facial action combinations during smiles

\begin{tabular}{ll} 
Consistent & \# of subjects* \\
Facial action combination & \\
\hline $\begin{array}{l}\text { Head movement during smile } \\
\text { Asymmetric motion during } \\
\text { smile }\end{array}$ & 7 \\
$\begin{array}{l}\text { Dampened smile (action units } \\
14,15,17)\end{array}$ & 6 \\
$\begin{array}{l}\text { Other consistent movements } \\
\text { All smiles include AU 6 }\end{array}$ & 4 \\
All smiles lack AU 6 & 12 \\
No pattern observed & 4 \\
$*$ some individuals listed in more than one category
\end{tabular}

Sixteen individuals showed either all Duchenne (AU 6+AU 12) smiles or all non-Duchenne (AU 12 only) smiles. Six individuals had consistently dampened smiles. Two individuals showed lip rearrangement, either through lip pressing motions (AU 24 and AU 28) or with tongue movements (AU 36 and AU 37) following the smile peak, and three subjects displayed marked asymmetry in facial actions, with one side consistently coded as acting with significantly greater intensity than the other.

An additional source of nonverbal individual difference was the regular presence of associated head movements during smiling in seven of the subjects studied. The most frequent head movements observed were AU 53 ("head up") and AUs 55 and 56 ("tilt head left" and tilt head right").

\subsection{Consistency in zygomaticus major muscle activity over time}

Additional information on stable individual differences was found in the EMG data, indirectly validating more qualitative results based on manual facial coding. When we compared the activity of zygomaticus major (z score relative to the mean raw zygomaticus major activity during baseline) among subjects, we found that it averaged 18.9 (SD 18.9) in the first session, and 14.3 (SD 20.1) while the same group watched the same comedy routine approximately one year later. The correlation for muscle activity between these two sessions was 0.578 ( $n=65$ subjects). There were no sex differences among subjects for these variables.

There is very little comparative data on within subject stability in affective (emotional) expression. One other study has shown that mother's expression of emotion remains stable over several years, and in the context of interaction with two different infants [7], although this study used manual coding rather than objective measures of facial activity.

\section{CONCLUSIONS}

Spontaneous smiles conform to some of the normative characteristics previously described in the literature. They are relatively fast in onset, averaging slightly over 0.5 seconds, when measured using automated feature point tracking. This figure is well within the range described for the onset of spontaneous smiles described elsewhere [1]. The most commonly observed smile types in this study were the non-Duchenne smile, with AU 12 only (32\%) and the Duchenne smile, with AU 12 followed shortly by AU 6 (32\%). Duchenne smiles in this sample were similar to smiles described previously [1]. Non-Duchenne smiles in the sample were equally frequent, however, and so may represent a naturally occurring variant of the smile of enjoyment - a smile of joy without the AU 6 marker. 
The timing of facial actions within multiple action unit smiles also appears to be a normative characteristic. A majority of spontaneous smiles showed other action units appearing either simultaneously, or following AU 12 within 30 frames (1 second). This characteristic of smiles differentiates them from other facial expressions such as the brow raise, the only other expression for which these dynamic characteristics has been reported.

Our results also indicated the possibility of stable individual differences in facial behavior. In the context of watching a comedy routine, we expected to see primarily Duchenne smiles, however, $16 \%$ of all smiles were dampened smiles. Because six subjects consistently displayed this type of smile, we suggest that in some cases, the dampened, or "miserable" smile may be a matter of individual style, rather than the expression of conflicting emotions. This stable individual difference, as well as others observed may indicate the presence of characteristic facial expressions in a large proportion of the population.

Facial EMG data provide additional support for individual stability over much longer time periods. The correlation of zygomaticus major activity between two sessions as long as a year apart suggests that individual patterns of facial behavior are stable. There are very little data on such long-term stability of expression. This study is only the second to demonstrate longterm stability in affective expression within subjects [7].

Although we anticipated sex differences in facial expression, there were no significant sex differences observed for consistency in action unit display, number of smiles observed (during the three jokes), or overall EMG activity.

These results have important implications for human computer interaction. For example, the construction of realistic representations of spontaneous human smiles should probably also include individuals whose smiles have these characteristics, even in the context of enjoyment. In addition, should patterns of expression prove to be characteristic of individuals, they may provide further identifying information for face recognition in naturalistic contexts.

\section{REFERENCES}

[1] Frank, M.G., Ekman, P. \& Friesen, W.V. "Behavioral Markers and Recognizability of the Smile of Enjoyment." Journal of Personality and Social Psychology, 64, 83-93, 1993.

[2] Grammer, K., Schiefenhovel, W., Schleidt, M., Lorenz, B. \& Eibl-Eibesfeldt, I. "Patterns on the Face: The Eyebrow Flash in Crosscultural Comparison”. Ethology, 77, 279-299, 1988.
[3] Ekman, P. Telling Lies: Clues to Deceit in the Marketplace, Politics, and Marriage. W.W. Norton, New York, 1985.

[4] Briton, N.J. \& Hall, J.A. "Gender-based Expectancies and Observer Judgments of Smiling”. Journal of Nonverbal Behavior, 19, 49-65, 1995.

[5] Ekman, P. \& Friesen, W.V. Facial Action Coding System. Consulting Psychologists Press, Palo Alto, 1978.

[6] Cohn, J.F., Zlochower, A., Lien, J. \& Kanade, T. “Automated Face Analysis by Feature Point Tracking has High Concurrent Validity with Manual FACS Coding”. Psychophysiology, 36, 3543, 1999.

[7] Moore, G.A., Cohn, J.F. \& S.B. Campbell "Mothers' affective behavior with infant siblings: Stability and change". Developmental Psychology, 33: 856-860, 1997.

[8]Tian, Y., Kanade, T.\& Cohn, J.F. "Recognizing Action Units for Facial Expression Analysis" IEEE Transactions on Pattern Analysis and Machine Intelligence, 23(2):97-115, 2001.

[9] Cacioppo, J. T., Petty, R. E., Losch, M. E., Kim, H.-S. "Electromyographic activity over facial muscle regions can differentiate the valence and intensity of affective reactions" Journal of Personality and Social Psychology,50(2):260-268, 1986.

\section{ACKNOWLEDGEMENTS}

This research was funded by NIH grant MH12579 to K. Schmidt and by NIH grant MH56193 and HID grant 29-203 to J. Cohn. We thank Bethany Peters and Rebecca McNutt for their assistance in the preparation of this paper. 